\title{
UNIQUENESS PROPERTIES OF THE INVARIATOR, LEADING TO SIMPLE COMPUTATIONS
}

\author{
LUIS M. CRUZ-ORIVE ${ }^{凶}$
}

Department of Mathematics, Statistics and Computation, Faculty of Sciences, University of Cantabria, Avda. Los Castros s/n, E-39005 Santander, Spain

e-mail: luis.cruz@unican.es

(Received October 26, 2011; revised February 7, 2012; accepted February 16, 2012)

\begin{abstract}
It is shown that, for a three dimensional particle (namely an arbitrary compact domain with piecewise smooth boundary in $\mathbb{R}^{3}$ ) the mean wedge volume defined on a given pivotal section is equal to the average nucleator estimator of the particle volume defined on that section. Further, if the particle is convex and it contains the pivotal point, then the flower area of a given pivotal section equals the average surfactor estimator defined on that section. These results are intended to throw some light on the standing conjecture that the functional defined on a pivotal section according to the invariator has a unique general expression. As a plus, the former result leads to a computational formula for the mean wedge volume of a convex polygon which is much simpler than the one published recently, and it is valid whether the fixed pivotal point is interior or exterior to the particle.
\end{abstract}

Keywords: flower area, invariator, nucleator, stereology, surface area, surfactor, volume, wedge volume.

\section{INTRODUCTION}

The invariator is a new stereological principle to generate a test line on an isotropic plane (called the pivotal plane) through a fixed point (called the pivotal point), which is effectively a test line with a motion invariant density in $\mathbb{R}^{3}$. This construction enjoys practical advantages because individual particle surface area and volume can be estimated from measurements made solely on a pivotal section (CruzOrive, 2005; Cruz-Orive et al., 2010). It can be shown that the volume of a particle in $\mathbb{R}^{3}$ is equal to $2 \pi$ times the mean volume of a three dimensional subset (called the wedge set) which is well defined on a pivotal section (Cruz-Orive, 2011, Eq. 18). If the particle is convex then its surface area is four times the mean flower area of a pivotal section (Cruz-Orive, 2005, Eq. 2.19).

Classical estimators of particle volume and surface area are the nucleator (Gundersen, 1988), and the surfactor (Jensen and Gundersen, 1987, 1989), respectively. For additional information on these estimators see Karlsson and Cruz-Orive (1997). In a recent generalization of the invariator the question has been raised of whether the invariator functional defined on a pivotal section to represent intrinsic particle volumes is unique (Gual-Arnau et al., 2010, Conjecture 4.1). If true, this conjecture would imply that, for any given pivotal plane, a suitable average of whatever estimator of an intrinsic volume would coincide with the corresponding invariator estimator.
The purpose of this paper is to prove the following results.

(i) For a particle in $\mathbb{R}^{3}$ the average of the nucleator formula on a pivotal section yields the mean wedge volume defined on that section (Proposition 1).

(ii) For a convex particle containing the pivotal point the average of the surfactor expression on a pivotal section coincides with the corresponding flower area. This result is proven separately for a convex particle with smooth boundary (Proposition 2), and for a convex polyhedron (Proposition 3).

The result (ii) was fortuitously found to hold for a ball in Cruz-Orive (2008) after Eq. 10.

As a plus, the following new results are also obtained.

(a) Proposition 1 leads to a computational formula for the mean wedge volume of a convex polygon (Corollary 1) which is much simpler than Eq. 61 from Cruz-Orive (2011). Moreover, the new formula is valid indistinctly for an interior or an exterior pivotal point.

(b) Proposition 3 leads to an alternative to Calka's formula (Cruz-Orive, 2011, Eq. 45) for the flower area of a convex polygon with respect to an interior pivotal point (Corollary 2).

Conclusions and open questions are presented in the final section. 


\section{EQUIVALENT \\ REPRESENTATIONS OF THE MEAN WEDGE VOLUME}

Consider a particle $Y \subset \mathbb{R}^{3}$, namely an arbitrary compact domain with nonempty interior $Y^{\circ}$ and piecewise smooth boundary $\partial Y$. Thus, $Y$ can be contained in a ball $B_{3}:=B_{3}(R)$ of finite radius $R$ and centred at a fixed point $O$, which is adopted as the pivotal point (Fig. 1, left).

\section{THE INVARIATOR CONSTRUCTION}

As in Cruz-Orive (2005, 2011, Section 2.1) let $L_{2}^{3}(0, t)$ represent a pivotal plane, namely an isotropic random plane through $O$ with normal direction $t \in \mathbb{S}_{+}^{2}$ which is uniform random over the unit hemisphere $\mathbb{S}_{+}^{2}$. If $t$ is parametrized by its spherical polar coordinates $(\phi, \theta), \phi \in[0,2 \pi), \theta \in[0, \pi / 2]$, then $\mathrm{d} t=\sin \theta \mathrm{d} \phi \mathrm{d} \theta$. With the notation adopted in the latter papers, the corresponding probability element is written,

$$
\mathbb{P}(\mathrm{d} t)=\frac{\mathrm{d} t}{2 \pi}, \quad t \in \mathbb{S}_{+}^{2} .
$$

In the pivotal equatorial disk $B_{2, t}:=B_{3} \cap L_{2}^{3}(0, t)$ choose a uniform random (UR) point $z$, with probability element

$$
\mathbb{P}(\mathrm{d} z \mid t)=\frac{\mathrm{d} z}{a}, \quad z \in B_{2, t},
$$

where $a$ denotes the area of $B_{2, t}$. Within the pivotal plane $L_{2}^{3}(0, t)$ draw a straight line $L_{1}^{2}(z ; t)$ through the point $z$ and normal to the axis joining $O$ and $z$. From Eqs. 1 and 2 , the randomness of $L_{1}^{2}(z ; t)$ is specified by the joint probability element

$$
\mathbb{P}(\mathrm{d} z, \mathrm{~d} t)=\frac{\mathrm{d} z}{a} \cdot \frac{\mathrm{d} t}{2 \pi}, \quad z \in B_{2, t}, t \in \mathbb{S}_{+}^{2} .
$$

The invariator principle states that the straight line $L_{1}^{2}(z ; t)$, called a $p$-line, is effectively isotropic uniform random (IUR) hitting the ball $B_{3}$.

\section{THE MEAN WEDGE VOLUME IS THE AVERAGED NUCLEATOR}

For convenience suppose that the particle $Y$ is included in a finite ball centred at $O$, that is $Y \subset$ $B_{3}$. Parametrize the $p$-line $L_{1}^{2}(z ; t)$ by its normal coordinates $(r, \omega),(r>0,0 \leq \omega<2 \pi)$, namely the polar coordinates of the point $z \in B_{2, t}$ with respect to a fixed reference half axis $O x_{1}$ in the pivotal plane. The volume of $Y$ can be expressed as follows,

$$
V(Y)=2 \pi \mathbb{E}_{t} \mathbb{E}_{\omega} V\{W(\omega ; t)\},
$$

where $W(\omega ; t)$ is the wedge solid defined on the pivotal section $Y \cap L_{2}^{3}(0, t)$ for each axis $L_{1}^{2}(0, \omega ; t)$ in that section, see Fig. 1, (for further details see Cruz-Orive, 2011, Section 2.3). On the other hand, $\mathbb{E}_{t}, \mathbb{E}_{\omega}$ denote expectations with respect to the probability elements given by Eq. 1 and $\mathbb{P}(\mathrm{d} \omega)=\mathrm{d} \omega /(2 \pi), \omega \in[0,2 \pi)$, respectively.

To simplify the notation suppose for the moment that $Y$ is star shaped with respect to an interior pivotal point $O$, which means that any ray joining $O$ with the boundary $\partial\left(Y \cap L_{2}^{3}(0 ; t)\right)$ of the pivotal section is connected. The length $\rho_{+}(\varphi ; t)$ of that ray is the radial function of the pivotal section. The nucleator representation of the volume of $Y$ reads,

$$
V(Y)=\frac{4 \pi}{3} \mathbb{E}_{t} \mathbb{E}_{\varphi}\left\{\rho_{+}^{3}(\varphi ; t)\right\},
$$

the inner expectation $\mathbb{E}_{\varphi}$ being with respect to $\mathbb{P}(\mathrm{d} \varphi)=\mathrm{d} \varphi /(2 \pi), \varphi \in[0,2 \pi)$. If $Y$ is not star shaped then it suffices to replace $\rho_{+}^{3}(\varphi ; t)$ with an alternating sum, see for instance Cruz-Orive (1987), Eq. B.4.

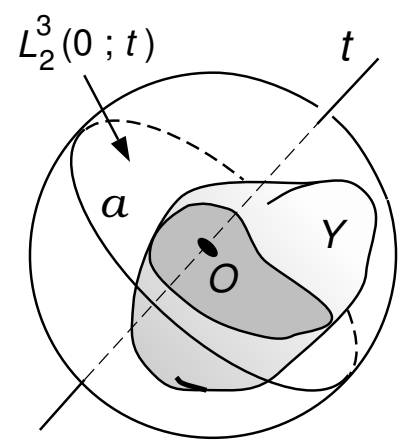

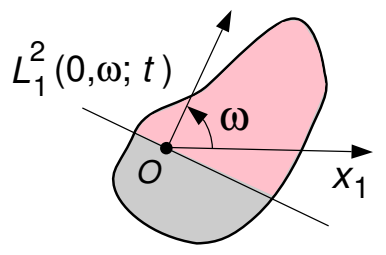

Pivotal section

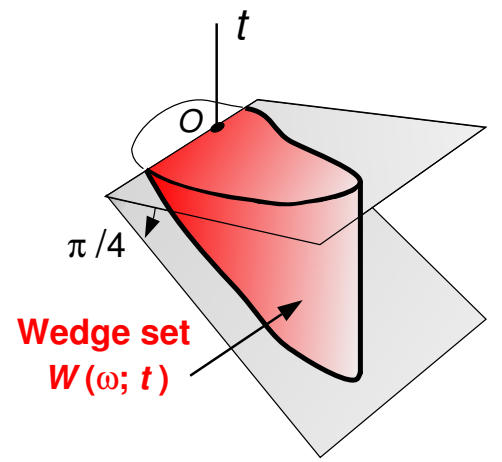

Fig. 1. Left: Particle $Y$ hit by a pivotal plane $L_{2}^{3}(0 ; t)$ of normal direction $t$. Middle and right: Pivotal section and the corresponding wedge set for a given orientation $\omega$. 
Proposition 1. For any pivotal section of a particle with respect to an interior pivotal point, namely for each orientation $t \in \mathbb{S}_{+}^{2}$ of the pivotal plane, the following identity holds,

$$
\mathbb{E}_{\omega} V\{W(\omega ; t)\}=\frac{2}{3} \mathbb{E}_{\varphi}\left\{\rho_{+}^{3}(\varphi ; t)\right\},
$$

that is, the mean wedge volume coincides with 2/3 times the averaged third power of the nucleator ray length.

Proof. For each $t \in \mathbb{S}_{+}^{2}$ and each $\omega \in[0,2 \pi)$ the wedge volume may be expressed as follows,

$$
V\{W(\omega ; t)\}=\int_{0}^{h(\omega ; t)} l(r, \omega ; t) r \mathrm{~d} r,
$$

where $l(r, \omega ; t)$ represents the total intercept length determined by the $p$-line in the pivotal section, see Fig. 2a. Further, $h(\omega ; t)$ is the upper bound of the distance $r$ for each $\omega$, and it coincides with the support function of the pivotal section when the latter is convex. Consider a point of unsigned abscissa $u$ along the $p$-line with respect to an arbitrary origin on this line. Then clearly,

$$
l(r, \omega ; t)=\int_{\left(Y \cap L_{2}^{3}(0, t)\right) \cap L_{1}^{2}(r, \omega ; t)} \mathrm{d} u,
$$

and the length elements $\mathrm{d} u$ and $\mathrm{d} r$ are orthogonal, see Fig. 2b. Therefore $\mathrm{d} x:=\mathrm{d} u \mathrm{~d} r$ represents an area element at a point $x$ in the pivotal plane. Let $(\rho, \varphi),(\rho>0,0 \leq \varphi<2 \pi)$ denote the polar coordinates of $x$ with respect to the fixed axis $O x_{1}$. Then $r=\rho|\cos (\omega-\varphi)|$ and it follows that,

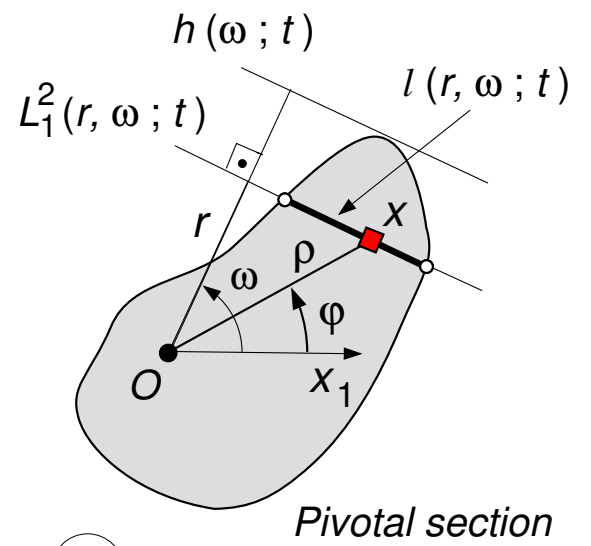

a

$$
\begin{aligned}
\mathbb{E}_{\omega} V & \{W(\omega ; t)\} \\
& =\frac{1}{2 \pi} \int_{0}^{\pi} \mathrm{d} \omega \int_{Y \cap L_{2}^{3}(0, t)} \rho|\cos (\omega-\varphi)| \mathrm{d} x \\
& =\frac{1}{2 \pi} \int_{Y \cap L_{2}^{3}(0, t)} \rho \mathrm{d} x \int_{0}^{\pi}|\cos (\omega-\varphi)| \mathrm{d} \omega \\
& =\frac{1}{\pi} \int_{Y \cap L_{2}^{3}(0, t)} \rho \mathrm{d} x \\
& =\frac{1}{\pi} \int_{0}^{2 \pi} \mathrm{d} \varphi \int_{0}^{\rho_{+}(\varphi ; t)} \rho^{2} \mathrm{~d} \rho \\
& =\frac{2}{3} \mathbb{E}_{\varphi}\left\{\rho_{+}^{3}(\varphi ; t)\right\} .
\end{aligned}
$$

In the first identity the range of integration of $\omega$ is $(0, \pi)$ instead of $(0,2 \pi)$ because the inner integral is extended over the whole pivotal section.

\section{ON THE COMPUTATION OF THE MEAN WEDGE VOLUME}

\section{COMPUTATION VIA THE INTEGRATED NUCLEATOR}

The third equality of Eq. 9 reads,

$$
\mathbb{E}_{\omega} V\{W(\omega ; t)\}=\frac{1}{\pi} \int_{Y \cap L_{2}^{3}(0, t)} \rho \mathrm{d} x .
$$

The right hand side of the preceding identity is essentially the integrated nucleator of Hansen et al. (2011). It was first presented in Jensen (1991) Eq. 5.5, see also Jensen and Rataj (2008), Section 5.1. Thus, for any pivotal section the mean wedge volume is equivalent to the integrated nucleator. Suppose that a pivotal section can be correctly segmented into $N$ pixels of area $a$, and let $\left\{d_{1}, d_{2}, \ldots, d_{N}\right\}$ denote the corresponding distances of the pixels from $O$. Then the

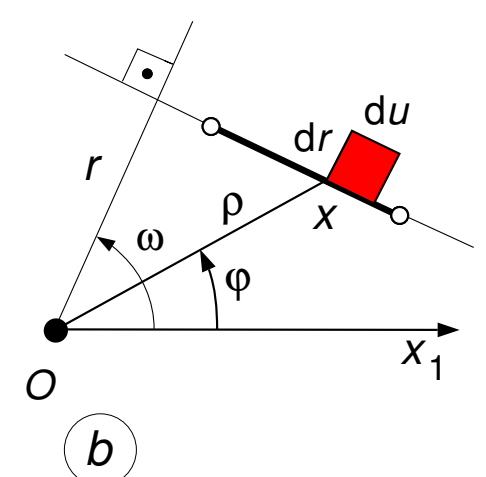

Fig. 2. Definitions used in the proof of Proposition 1. (a) Pivotal section hit by a p-line $L_{1}^{2}(r, \omega ; t)$. (b) Details used in the text. 
estimator of the mean wedge volume can be computed as,

$$
\hat{\mathbb{E}}_{\omega} V\{W(\omega ; t)\}=\frac{a}{\pi} \sum_{i=1}^{N} d_{i},
$$

and the corresponding estimator of the particle volume reads,

$$
\hat{V}(Y)=2 a \sum_{i=1}^{N} d_{i}
$$

which is a discretised version of the first integral in Section 2.1.2 of Hansen et al. (2011).

In Cruz-Orive (2011), Section 6.4, an exact computational formula is given for the mean wedge volume when a convex pivotal section is approximated by a convex polygon of $n$ vertexes. The idea is that, if the pivotal section cannot be segmented, then at least a few 'evenly spaced' points may be selected along its boundary, either automatically, or semiautomatically with the aid of a cursor.

\section{COMPUTATION OF THE MEAN WEDGE VOLUME DEFINED ON A CONVEX POLYGON VIA THE AVERAGED NUCLEATOR: A VAST IMPROVEMENT ON CRUZ-ORIVE (2011)}

The mean wedge volume formulae for a convex polygon given by Eq. 61 and Eq. 80 in Cruz-Orive (2011) are unnecessarily complicated and do not hold for an exterior pivotal point. By virtue of Eq. 6 it suffices to integrate the third power of the radius vector of the polygon, which is much simpler than the direct computation adopted in the latter paper. The corresponding formula is given in Corollary 1 below. For completeness, the pertinent notation from Section 5.2 of Cruz-Orive (2011) is given next.

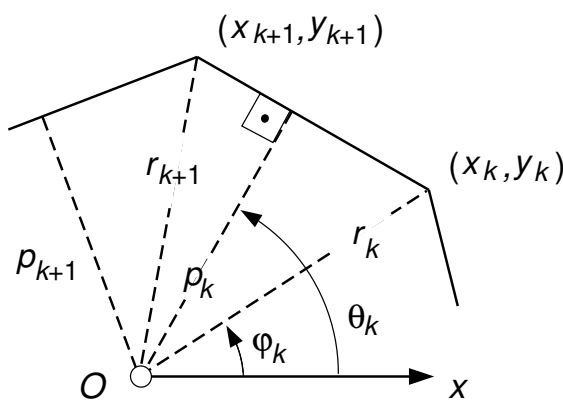

Fig. 3. A portion of a convex polygon describing the notation used in Corollary 1.

Prerequisites. A pivotal section $Y \cap L_{2}^{3}(0, t)$ of a convex particle $Y$ is almost surely convex and can be approximated by a convex $n$-gon $K \subset \mathbb{R}^{2}$. To follow the ensuing development it is convenient to suppose that
$O \in K^{\circ}$, but Corollary 1 does not require this. However, if $O \notin K^{\circ}$ then the rectangular reference frame $O x y$ has to be chosen so that $K$ lies in the upper half plane. The following elements are needed, see Fig. 3.

- The rectangular coordinates of the vertices are given by the counterclockwise cyclic sequence $\left\{\left(x_{1}, y_{1}\right),\left(x_{2}, y_{2}\right), \ldots,\left(x_{n}, y_{n}\right)\right\}$, with $\left(x_{n+1}, y_{n+1}\right)=$ $\left(x_{1}, y_{1}\right)$. It is assumed that $\left(x_{1}, y_{1}\right)$ is the rightmost vertex, namely

$$
x_{1}=\sup \left\{x_{1}, x_{2}, \ldots, x_{n}\right\} .
$$

- Polar coordinates of the vertices: $\left\{\left(r_{1}, \varphi_{1}\right)\right.$, $\left.\left(r_{2}, \varphi_{2}\right), \ldots,\left(r_{n}, \varphi_{n}\right)\right\}$, with $\varphi_{n+1}=\varphi_{1}+2 \pi$. Note that the sequence $\left\{\varphi_{1}, \varphi_{2}, \ldots, \varphi_{n}\right\}$ is monotonically non decreasing if $O \in K^{\circ}$, but not otherwise.

- Normal coordinates of the straight lines containing the sides: $\left\{\left(p_{1}, \theta_{1}\right),\left(p_{2}, \theta_{2}\right), \ldots,\left(p_{n}, \theta_{n}\right)\right\}$. The 'apothems' $\left\{p_{i}\right\}$ are all positive if $O \in K^{\circ}$, but not otherwise. The condition that the first vertex is the rightmost one implies that:

$$
0 \leq \theta_{1}<\theta_{2}<\cdots<\theta_{n}<\theta_{1}+2 \pi .
$$

Next the relevant connections are displayed. Define the function

$$
\varphi(x, y)= \begin{cases}\cos ^{-1}\left(x / \sqrt{x^{2}+y^{2}}\right) & \text { if } y \geq 0 \\ 2 \pi-\cos ^{-1}\left(x / \sqrt{x^{2}+y^{2}}\right) & \text { if } y<0 .\end{cases}
$$

Then, for $k=1,2, \ldots, n$,

$$
\begin{aligned}
r_{k} & =\left(x_{k}^{2}+y_{k}^{2}\right)^{1 / 2}, \\
\varphi_{k} & =\varphi\left(x_{k}, y_{k}\right), \\
\theta_{k} & =\varphi\left(y_{k+1}-y_{k},-\left(x_{k+1}-x_{k}\right)\right), \\
p_{k} & =r_{k} \cos \left(\theta_{k}-\varphi_{k}\right) .
\end{aligned}
$$

Corollary 1. For a convex $n$-gon $K \subset \mathbb{R}^{2}$ the mean volume of the wedge set $W_{K}(\omega)$ defined on $K$ with respect to an arbitrary pivotal point in its plane may be expressed as follows,

$$
2 \pi \mathbb{E}_{\omega} V\left\{W_{K}(\omega)\right\}=\frac{1}{3} \sum_{k=1}^{n} p_{k}^{3} \cdot \psi\left(\varphi_{k}, \varphi_{k+1}, \theta_{k}\right),
$$

where

$$
\begin{aligned}
\psi\left(\omega_{1}, \omega_{2}, \theta\right) & \\
:= & \frac{\sin \left(\omega_{2}-\theta\right)}{\cos ^{2}\left(\omega_{2}-\theta\right)}-\frac{\sin \left(\omega_{1}-\theta\right)}{\cos ^{2}\left(\omega_{1}-\theta\right)} \\
& +\log \left(\frac{\tan \left(\left(\omega_{2}-\theta\right) / 2+\pi / 4\right)}{\tan \left(\left(\omega_{1}-\theta\right) / 2+\pi / 4\right)}\right) .
\end{aligned}
$$

If $O \notin K^{\circ}$, then $K$ must be contained in the upper half plane. 
Proof. For $k=1,2, \ldots, n$ let $\rho_{k}(\omega), \omega \in\left[\varphi_{k}, \varphi_{k+1}\right)$ denote the radial function of $K$. Bearing in mind that $\rho_{k}(\omega) \cos \left(\omega-\theta_{k}\right)=p_{k}$, by virtue of Proposition 1 one has,

$$
\begin{aligned}
2 \pi \mathbb{E}_{\omega} V & \left\{W_{K}(\omega)\right\} \\
= & \frac{2}{3} \sum_{k=1}^{n} \int_{\varphi_{k}}^{\varphi_{k+1}} \rho_{k}^{3}(\omega) \mathrm{d} \omega \\
= & \frac{2}{3} \sum_{k=1}^{n} p_{k}^{3} \int_{\varphi_{k}}^{\varphi_{k+1}} \frac{\mathrm{d} \omega}{\cos ^{3}\left(\omega-\theta_{k}\right)} .
\end{aligned}
$$

On the other hand,

$$
\int \frac{\mathrm{d} x}{\cos ^{3} x}=\frac{1}{2} \frac{\sin x}{\cos ^{2} x}+\frac{1}{2} \log \tan \left(\frac{x}{2}+\frac{\pi}{4}\right)+C,
$$

whereby Corollary 1 follows.

\section{COMPUTATION OF THE MEAN WEDGE VOLUME WITH THE NEW FORMULA: EXAMPLES}

To check Eq. 17 I have resorted to the model used in Section 8.1 of Cruz-Orive (2011) in which the particle $Y \subset \mathbb{R}^{3}$ is a finite ball.

Case of an interior pivotal point, $O \in Y^{\circ}$. Here a pivotal section is almost surely a disk containing $O$, (Fig. 4). Let $r, l$ denote the section radius and the distance of $O$ from the section centre, respectively. Setting $\lambda:=l / r \in(0,1)$, the exact invariator estimator of the particle volume is,

$$
\begin{aligned}
2 \pi \mathbb{E}_{\omega} & V\{W(\omega)\} \\
& =\frac{8}{9} r^{3}\left[\left(7+\lambda^{2}\right) E\left(\lambda^{2}\right)-4\left(1-\lambda^{2}\right) K\left(\lambda^{2}\right)\right],
\end{aligned}
$$

(Cruz-Orive, 2011), where $K, E$ represent the complete elliptic integrals of the first and second kinds, respectively. Retaining 5 exact digits, the numerical values of the preceding expression for $\lambda=0.1,0.3$, 0.70 .9 and $r=1$ are 4.2201, 4.4699, 5.6788, 6.5935, respectively. Approximating the disk by a regular polygon of $n=1200$ vertices, formula 17 returns the 5 exact digits in $2.0 \mathrm{~s}$ computing time on a SunBlade 1500 workstation. The corresponding time with the earlier exact formula 61 of Cruz-Orive (2011) is $10.0 \mathrm{~s}$.

Case of an exterior pivotal point, $O \notin Y^{\circ}$. Whenever $Y \cap L_{2}^{3}(0, t) \neq \emptyset$, the pivotal section is a disk. Let $r, l$ stand as above, and set $\lambda:=l / r>1$. Then, conditional on the hitting event, (Fig. 5),

$$
\begin{aligned}
2 \pi \mathbb{E}_{\omega} V & \{W(\omega)\} \\
= & \frac{8 r^{3}}{9 \lambda}\left[\lambda^{2}\left(7+\lambda^{2}\right) E\left(\lambda^{-2}\right)\right. \\
& \left.-\left(\lambda^{2}+3\right)\left(\lambda^{2}-1\right) K\left(\lambda^{-2}\right)\right] .
\end{aligned}
$$

The preceding result is derived in the Appendix. Retaining 5 exact digits, the numerical values of the preceding expression for $\lambda=1.5,2.0,2.5,3.0$ and $r=1$ are $9.9590,12.963,16.024,19.112$, respectively. Approximating the disk by a regular polygon of $n=$ 2000 vertices, formula 17 returns the 5 exact digits in 3.0 s computing time. Eq. 61 and Eq. 80 of Cruz-Orive (2011) are not valid in this case.

\section{THE INTEGRATED SURFACTOR IS THE FLOWER AREA}

For a convex particle $Y$ containing the pivotal point, the invariator principle implies that the particle surface area satisfies the identity,

$$
\begin{aligned}
S(\partial Y) & =4 \mathbb{E}_{t}\left\{A\left(H_{t}\right)\right\} \\
& =2 \mathbb{E}_{t}\left\{\int_{0}^{2 \pi} h^{2} \mathrm{~d} \omega\right\},
\end{aligned}
$$

(Cruz-Orive, 2005) where $H_{t}$ represents the support set or 'flower' of a pivotal section $Y \cap L_{2}^{3}(0, t)$ with respect to the pivotal point $O \in Y^{\circ}$, and $h:=h(\omega),(0 \leq$ $\omega<2 \pi)$ denotes the support function of the pivotal section, namely the radial function of the flower. Recall that $\partial H_{t}$ is the locus of the foot $P(h, \omega)$ of the

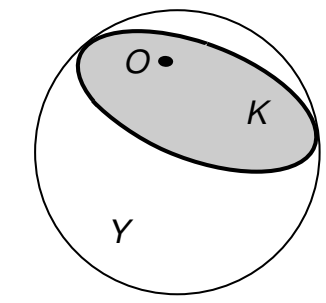

Ball and pivotal section

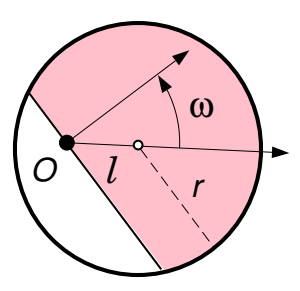

Pivotal section

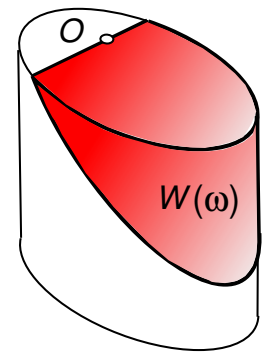

Wedge

Fig. 4. The wedge set of a pivotal section of a ball with an interior pivotal point $O$. 


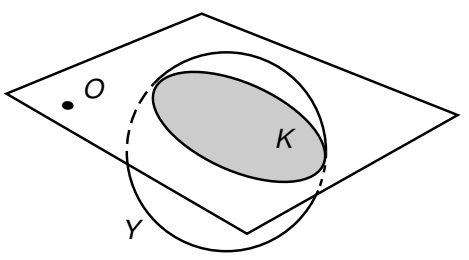

Ball and pivotal section
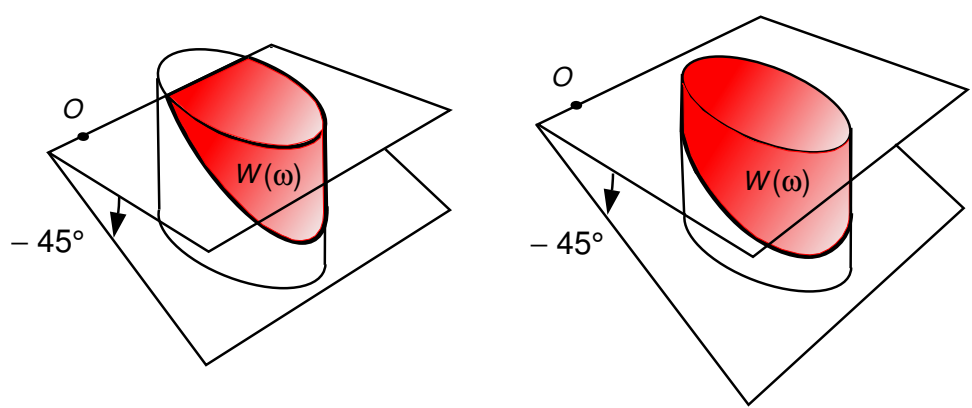

Wedges with exterior pivotal point $O$

Fig. 5. The wedge set of a pivotal section of a ball with an exterior pivotal point $O$.

perpendicular from $O$ to the tangent to $\partial\left(Y \cap L_{2}^{3}(0, t)\right)$ at a point $M(\rho, \varphi)$, see Fig. 6 a.

Alternatively, the surfactor representation of the particle surface area is,

$$
\begin{aligned}
S(\partial Y) & =4 \pi \mathbb{E}_{t} \mathbb{E}_{\varphi}\left\{\rho^{2}(1+\alpha \tan \alpha)\right\} \\
& =2 \mathbb{E}_{t}\left\{\int_{0}^{2 \pi} \rho^{2}(1+\alpha \tan \alpha) \mathrm{d} \varphi\right\}
\end{aligned}
$$

(Jensen and Gundersen, 1987, 1989), where $\rho=\rho(\varphi)$, $(0 \leq \varphi<2 \pi)$ represents the radial function of the pivotal section. The expectation $\mathbb{E}_{\varphi}$ is with respect to the uniform probability element $\mathbb{P}(\mathrm{d} \varphi)=\mathrm{d} \varphi /(2 \pi)$. Further, $\alpha \in[-\pi / 2, \pi / 2]$ is the angle between the normal to $\partial\left(Y \cap L_{2}^{3}(0, t)\right)$ at a point $M(\rho, \varphi)$ and an axis joining this boundary point with $O$, see Fig. 6 a. Note that $\alpha=\varphi-\omega$.

The purpose of this section is to show that, for each orientation $t \in \mathbb{S}^{2}$ of the pivotal plane, the expressions in curly brackets in the right hand sides of Eq. 23 and Eq. 24 coincide. This is done first for a strictly

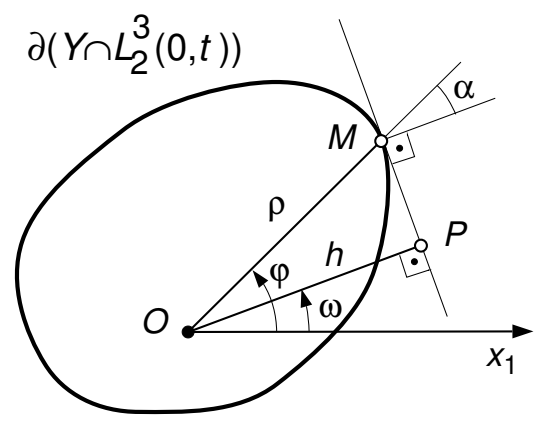

a convex particle with smooth boundary (Proposition 2) and then for a convex polyhedral particle (Proposition $3)$.

\section{CASE OF A CONVEX PARTICLE WITH SMOOTH BOUNDARY}

Here the particle $Y$ is assumed to be strict convex with a smooth boundary $\partial Y$ of class $C^{2}$. Thus, $\partial Y$ contains no flat faces and no corners, and has a unique tangent plane at each point. Moreover the pivotal point lies in the interior of the particle, namely $O \in Y^{\circ}$.

Proposition 2. For each pivotal section of a strict convex and smooth particle with boundary of class $C^{2}$ containing the pivotal point, the following identity holds,

$$
\int_{0}^{2 \pi} \rho^{2}(1+\alpha \tan \alpha) \mathrm{d} \varphi=\int_{0}^{2 \pi} h^{2} \mathrm{~d} \omega .
$$

The result holds trivially when the particle is a ball with the pivotal point at its centre, because in this case
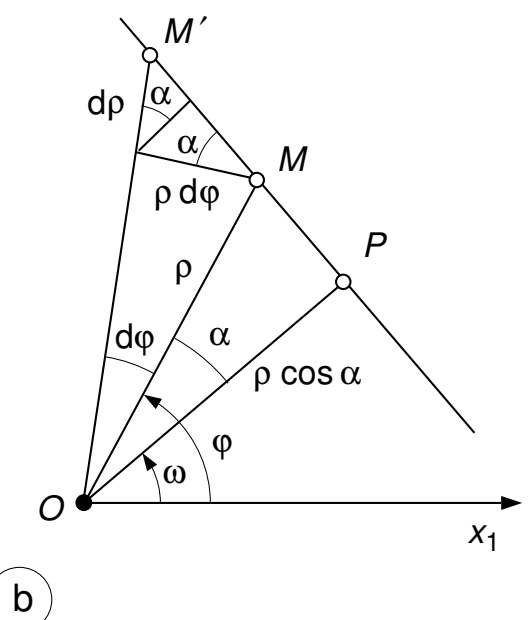

Fig. 6. Geometrical parameters used in the proof of Proposition 2. (a) Pivotal section of a convex particle Y with interior pivotal point $O$. (b) Details. 
$\alpha=0$ whereas $\rho$ and $h$ are constant and equal to the radius of the ball. In the sequel it is assumed that $\alpha \neq 0$ almost surely in a range of $\varphi$ of positive measure.

To simplify the proof I first establish Lemma 1 below which holds for a planar set $K$ which may be regarded as a pivotal section of $Y$. The radial functions of $K$ and of the flower $H_{K}$ of $K$ with respect to a fixed axis $O x_{1}$ are denoted by $\rho(\varphi)$ and $h(\omega)$ respectively. Recall that,

$$
\begin{aligned}
A(K) & =\frac{1}{2} \int_{0}^{2 \pi} \rho^{2} \mathrm{~d} \varphi, \\
A\left(H_{K}\right) & =\frac{1}{2} \int_{0}^{2 \pi} h^{2} \mathrm{~d} \omega .
\end{aligned}
$$

Remark 1. Given the polar angle $\varphi \in[0,2 \pi)$ of a point of $\partial K$, the angles $\alpha$ and $\omega$ can be expressed as functions of $\varphi$ only. Likewise, given the polar angle $\omega \in[0,2 \pi)$ of a point of $\partial H_{K}$, the angles $\alpha$ and $\varphi$ can be expressed as functions of $\omega$ only. Thus, an integral with respect to $\mathrm{d} \alpha$, for instance, can be interpreted as an integral with respect to $\mathrm{d} \alpha(\varphi)$, or to $\mathrm{d} \alpha(\omega)$, over the interval $[0,2 \pi)$.

Lemma 1. The flower area $A\left(H_{K}\right)$ of a planar, strict convex and smooth set $K$ with boundary of class $C^{2}$ and containing the origin may be expressed as follows,

$$
A\left(H_{K}\right)=A(K)+\frac{1}{2} \int_{0}^{2 \pi} \rho^{2} \sin ^{2} \alpha \mathrm{d} \omega,
$$

where the angle $\alpha$ is defined as in Eq. 24. Equivalently,

$$
\int_{0}^{2 \pi} h^{2} \mathrm{~d} \omega=\int_{0}^{2 \pi} \rho^{2} \mathrm{~d} \varphi+\int_{0}^{2 \pi} \rho^{2} \sin ^{2} \alpha \mathrm{d} \omega .
$$

Heuristic proof of Lemma 1. If Lemma 1 holds, then the second term in the right hand side of Eq. 27 is the area of the portion of $H_{K}$ comprised between the two boundaries $\partial K$ and $\partial H_{K}$, namely the area of the proper set difference $D_{K}:=H_{K} \backslash K$, see Fig. 7. By construction, the region $D_{K}$ is swept by the straight line segment $M P$, where the endpoint $M$ moves along $\partial K$ whereas the other endpoint $P$ describes $\partial H_{K}$. The segment $M P$ is contained in the tangent to $\partial K$ at $M$, and $P$ is the foot of the perpendicular from $O$ to that tangent. As the point $M$ moves along an elementary arc of $\partial K$ into $M^{\prime}$, the point $P$ moves along a corresponding elementary arc of $\partial H_{K}$ into $P^{\prime}$. Thus the segment $M P$ sweeps an elementary triangle of area

$$
\frac{1}{2}|M P|^{2} \mathrm{~d} \omega=\frac{1}{2} \rho^{2} \sin ^{2} \alpha \mathrm{d} \omega,
$$

from which Eq. 27 follows.

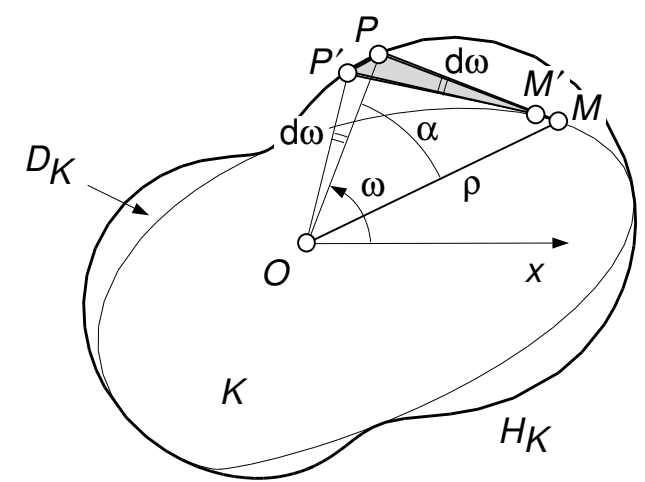

Fig. 7. Definitions used in the heuristic proof of Lemma 1.

Proof of Lemma 1. The ordinary area element $\mathrm{d} A$ in polar coordinates is $(1 / 2) \rho^{2} \mathrm{~d} \varphi$. However, for the present purposes a different representation is needed. The area element may be regarded as an elementary triangle $O M M^{\prime}$ of height $\rho \cos \alpha$ and base $\sin \alpha \mathrm{d} \rho+$ $\rho \cos \alpha \mathrm{d} \varphi$, see Fig. 6b. Therefore,

$$
\begin{aligned}
\mathrm{d} A & =\frac{1}{2} \rho \sin \alpha \cos \alpha \mathrm{d} \rho+\frac{1}{2} \rho^{2} \cos ^{2} \alpha \mathrm{d} \varphi \\
& =\frac{1}{2} \rho \rho_{\alpha}^{\prime} \sin \alpha \cos \alpha \mathrm{d} \alpha+\frac{1}{2} \rho^{2} \cos ^{2} \alpha \mathrm{d} \varphi,
\end{aligned}
$$

where $\rho_{\alpha}^{\prime}:=\mathrm{d} \rho / \mathrm{d} \alpha$. Thus,

$2 A(K)=\int_{0}^{2 \pi} \rho \rho_{\alpha}^{\prime} \sin \alpha \cos \alpha \mathrm{d} \alpha+\int_{0}^{2 \pi} \rho^{2} \cos ^{2} \alpha \mathrm{d} \varphi$,

where, by virtue of Remark $1, \mathrm{~d} \alpha$ may be interpreted as $\alpha_{\varphi}^{\prime} \mathrm{d} \varphi$. Integration by parts with $u=\sin \alpha \cos \alpha$ and $\mathrm{d} v=\rho \rho_{\alpha}^{\prime} \mathrm{d} \alpha$ yields,

$$
\begin{aligned}
\int_{0}^{2 \pi} \rho \rho_{\alpha}^{\prime} & \sin \alpha \cos \alpha \mathrm{d} \alpha \\
& =-\frac{1}{2} \int_{0}^{2 \pi} \rho^{2}\left(\cos ^{2} \alpha-\sin ^{2} \alpha\right) \mathrm{d} \alpha .
\end{aligned}
$$

Recalling that $\alpha=\varphi-\omega$ and $\rho \cos \alpha=h$, simplification of the right hand side of Eq. 31 leads to Eq. 28.

Proof of Proposition 2. From Fig. 6b one obtains the key relation

$$
\tan \alpha=\frac{\mathrm{d} \rho}{\rho \mathrm{d} \varphi}=\frac{\rho_{\varphi}^{\prime}}{\rho}
$$

Therefore,

$$
\int_{0}^{2 \pi} \rho^{2} \alpha \tan \alpha \mathrm{d} \varphi=\int_{0}^{2 \pi} \rho \rho_{\varphi}^{\prime} \alpha \mathrm{d} \varphi .
$$


Integration by parts with $u=\alpha$ and $\mathrm{d} v=\rho \rho_{\varphi}^{\prime} \mathrm{d} \varphi$ yields,

$$
\begin{aligned}
\int_{0}^{2 \pi} \rho^{2} \alpha \tan \alpha \mathrm{d} \varphi \\
=-\frac{1}{2} \int_{0}^{2 \pi} \rho^{2} \mathrm{~d} \alpha \\
=-\frac{1}{2} \int_{0}^{2 \pi} \rho^{2} \mathrm{~d} \varphi \\
\quad+\frac{1}{2} \int_{0}^{2 \pi} \rho^{2}\left(\sin ^{2} \alpha+\cos ^{2} \alpha\right) \mathrm{d} \omega .
\end{aligned}
$$

Bearing in mind that $\rho \cos \alpha=h$, substituting the preceding result into the left hand side of Eq. 25 and applying Lemma 1, the right hand side of Eq. 25 is obtained.

\section{CASE OF A CONVEX POLYHEDRAL PARTICLE}

Proposition 2 has been proved under the assumption that the particle $Y$ is strict convex and it has a unique tangent plane at every point of its boundary. Because this property is rather restrictive (for instance it excludes polyhedra), here it is assumed that $Y$ is a convex polyhedron. Thus, every pivotal section is almost surely a convex polygon.

Proposition 3. Eq. 25 holds also for any convex n-gon with an interior pivotal point.

Proof. Based on the preliminary results and notation leading to Corollary 1 , for a convex $n$-gon the left hand side of Eq. 25 may be written as follows,

$$
\begin{aligned}
\int_{0}^{2 \pi} \rho^{2}(1+\alpha \tan \alpha) \mathrm{d} \varphi \\
=\sum_{k=1}^{n} \int_{\varphi_{k}}^{\varphi_{k+1}} \frac{p_{k}^{2}}{\cos ^{2} \alpha}(1+\alpha \tan \alpha) \mathrm{d} \varphi
\end{aligned}
$$

where $\alpha:=\varphi-\theta_{k}$ in the preceding integrand, hence $\mathrm{d} \varphi=\mathrm{d} \alpha$. On the other hand, by Calka's formula (Cruz-Orive, 2011, Eq. 45), the right hand side of Eq. 25 is,

$$
\begin{aligned}
& \int_{0}^{2 \pi} h^{2} \mathrm{~d} \omega \\
& \quad=\frac{1}{4} \sum_{k=1}^{n} r_{k+1}^{2}\left[\zeta\left(\theta_{k+1}-\varphi_{k+1}\right)-\zeta\left(\theta_{k}-\varphi_{k+1}\right)\right], \\
& \zeta(x):=2 x+\sin (2 x) .
\end{aligned}
$$

Thus, the right hand sides of Eq. 36 and Eq. 37 must coincide. This is readily verified on substituting the following results

$$
\begin{aligned}
& \int \frac{1+x \tan x}{\cos ^{2} x} \mathrm{~d} x=\frac{1}{2} \tan x+\frac{1}{2} \frac{x}{\cos ^{2} x}+C, \\
& p_{k}=r_{k} \cos \left(\theta_{k}-\varphi_{k}\right)=r_{k+1} \cos \left(\theta_{k}-\varphi_{k+1}\right),
\end{aligned}
$$

into the right hand side of Eq. 36, and simplifying.

\section{AN ALTERNATIVE TO CALKA'S FORMULA FOR THE FLOWER AREA OF A CONVEX POLYGON}

Corollary 2. The flower area of a convex $n$-gon $K$ may be expressed as follows,

$$
\begin{aligned}
& A\left(H_{K}\right)=\frac{1}{4} \sum_{k=1}^{n} p_{k}^{2}\left[\gamma\left(\varphi_{k+1}-\theta_{k}\right)-\gamma\left(\varphi_{k}-\theta_{k}\right)\right], \\
& \gamma(x):=\tan x+\frac{x}{\cos ^{2} x} .
\end{aligned}
$$

Proof. Bearing the first Eq. 38 in mind the result is immediate because by Proposition 3 the right hand side of Eq. 36 is equal to $2 A\left(H_{K}\right)$.

\section{CONCLUSIONS AND COMMENTS}

Propositions 1 and 2 throw some light on the uniqueness conjecture mentioned in the Introduction. However, a full proof of the conjecture for $\mathbb{R}^{3}$ would still require that, apart from the invariator estimators, no other local estimators exist but the nucleator and the surfactor.

Proposition 2 holds for a convex particle with an interior pivotal point. For an exterior pivotal point the right hand side of Eq. 25 is not pertinent because in this case the flower area of a convex set is not the second Eq. 26 but

$$
A\left(H_{K}\right)=\frac{1}{2} \int_{0}^{2 \pi} h(\omega) \cdot|h(\omega)| \mathrm{d} \omega .
$$

Note also that when $O \notin Y^{\circ}$ the left hand side of Eq. 25 may not exist because the angle $\alpha$ will be equal to $\pi / 2$ at least for the two tangent axes to the section boundary from $O$. Likewise, if the pivotal section is a convex polygon then the support line of one or two sides may pass through $O$, with the same consequence.

Corollary 1, (Eq. 17), constitutes a vast improvement over Eq. 61 of Cruz-Orive (2011). In that paper I completely overlooked the key equivalence (Eq. 6), which was already explicit in Gual-Arnau et al. (2010), Section 4.5. Moreover, due to the fact that the $\left\{p_{k}\right\}$ are signed Eq. 17 holds also for an exterior pivotal point. 
Eq. 39 may be an interesting alternative to Calka's formula, but it does not seem to enjoy any particular computational advantage over the latter. Further, for an exterior pivotal point, a formula for the flower area of a convex polygon seems to require a separate treatment (in preparation).

As pointed out by an anonymous referee, Proposition 1 can be obtained as a particular case of Proposition 2 from Auneau and Jensen (2010). Likewise, Propositions 2 and 3 can be condensed into a single proposition valid for convex particles with piecewise smooth boundary; this would require some elaboration of Proposition 4 of the latter paper together with some general results from the book of Jensen (1998). Here I have chosen to concentrate on relevant particular cases in $\mathbb{R}^{3}$ using direct elementary proofs. Moreover Proposition 3 is useful because it leads to Corollary 2 directly; in general, separate results for convex polygons are useful because they constitute the basis of practical approximation formulae for convex particles.

\section{ACKNOWLEDGMENTS}

I wish to thank two anonymous referees for carefully reading the original manuscript and pointing out a number suggestions for improvement. One of them prompted me to add the last paragraph of the Conclusions section, and the appendix. Work supported by the Spanish Ministry of Education and Science I+D Project MTM-2009-14500-C02-01.

\section{REFERENCES}

Abramowitz M, Stegun IA (1965). Handbook of Mathematical Functions. New York: Dover.

Auneau J, Jensen EVB (2010). Expressing intrinsic volumes as rotational integrals. Adv Appl Math 45:1-11.

Cruz-Orive LM (1987). Particle number can be estimated using a disector of unknown thickness: the selector. J Microsc 145:121-42. .

Cruz-Orive LM (2005). A new stereological principle for test lines in 3D. J Microsc 219:18-28.

Cruz-Orive LM (2008). Comparative precision of the pivotal estimators of particle size. Image Anal Stereol 27:17-22.

Cruz-Orive LM (2011). Flowers and wedges for the stereology of particles. J Microsc 243:86-102.

Cruz-Orive LM, Ramos-Herrera ML, Artacho-Pérula E (2010). Stereology of isolated objects with the invariator. J Microsc 240:94-110.

Gundersen HJG (1988). The nucleator. J Microsc 151:3-21.
Gual-Arnau X, Cruz-Orive LM, Nuño-Ballesteros JJ (2010). A new rotational integral formula for intrinsic volumes in space forms. Adv Appl Math 44:298-308.

Hansen LV, Nyengaard JR, Andersen JB, Jensen EBV (2011). The semi-automatic nucleator. J Microsc 242:206-15.

Jensen EBV (1991). Recent developments in the stereological analysis of particles. Ann Inst Statist Math 43:455-68.

Jensen EBV (1998). Local Stereology. Singapore: World Scientific.

Jensen EB, Gundersen HJG (1987). Stereological estimation of surface area of arbitrary particles. Acta Stereol 6 (Suppl III):25-30.

Jensen EB, Gundersen HJG (1989). Fundamental stereological formulae based on isotropically orientated probes through fixed points with applications to particle analysis. J Microsc 153:249-67.

Jensen EBV, Rataj J (2008). A rotational integral formula for intrinsic volumes. Adv Appl Math 41:530-60.

Karlsson LM, Cruz-Orive LM (1997). Estimation of mean particle size from single sections. J Microsc 186:121-32.

\section{APPENDIX: MEAN WEDGE VOLUME FOR A DISK WITH EXTERIOR PIVOTAL POINT}

Our purpose is to derive Eq. 22. With reference to Fig. 8, Eq. 7 yields,

$$
\begin{aligned}
\pi \mathbb{E}_{\omega} V & \{W(\omega)\} \\
= & \int_{\omega_{0}}^{\omega_{1}} \mathrm{~d} \omega \int_{0}^{r-l \cos \omega} 2 \rho \sqrt{r^{2}-(\rho+l \cos \omega)^{2}} \mathrm{~d} \rho \\
& +\int_{\omega_{1}}^{\pi} \mathrm{d} \omega \int_{-r-l \cos \omega}^{r-l \cos \omega} 2 \rho \sqrt{r^{2}-(\rho+l \cos \omega)^{2}} \mathrm{~d} \rho
\end{aligned}
$$

where $\omega_{0}=\pi / 2-\sin ^{-1}(r / l), \omega_{1}=\pi / 2+\sin ^{-1}(r / l)$, $0<r<l<\infty$. The symbol $t$ has been omitted in the left hand side. After some algebra assisted by Mathematica ${ }^{\circledR}$, the following result is obtained,

$$
\begin{aligned}
\pi \mathbb{E}_{\omega} V\{W(\omega)\} & \\
= & \frac{8}{9} l^{3} \lambda\left[\left(1+7 \lambda^{2}\right) E\left(\sin ^{-1} \lambda, \lambda^{-2}\right)\right. \\
& \left.+4\left(1-\lambda^{2}\right) F\left(\sin ^{-1} \lambda, \lambda^{-2}\right)\right],
\end{aligned}
$$

where $\lambda:=r / l \in(0,1)$, and $F(\phi, m), E(\phi, m)$ denote the incomplete elliptic integrals of the first and second kind, respectively. A more convenient expression depending on the complete elliptic integrals $K, E$ of the first and second kind, respectively, can be obtained by 
means of the following identities, valid for $\lambda \in(0,1)$,

$$
\begin{aligned}
E\left(\sin ^{-1} \lambda, \lambda^{-2}\right) & =\int_{0}^{\sin ^{-1} \lambda} \sqrt{1-\lambda^{-2} \sin ^{2} x} \mathrm{~d} x \\
& =\lambda \int_{0}^{\pi / 2} \frac{\cos ^{2} x \mathrm{~d} x}{\sqrt{1-\lambda^{2} \sin ^{2} x}} \\
& =\lambda^{-1}\left[E\left(\lambda^{2}\right)-\left(1-\lambda^{2}\right) K\left(\lambda^{2}\right)\right] \\
F\left(\sin ^{-1} \lambda, \lambda^{-2}\right) & =\int_{0}^{\sin ^{-1} \lambda} \frac{\mathrm{d} x}{\sqrt{1-\lambda^{-2} \sin ^{2} x}} \\
& =\lambda \int_{0}^{\pi / 2} \frac{\mathrm{d} x}{\sqrt{1-\lambda^{2} \sin ^{2} x}} \\
& =\lambda K\left(\lambda^{2}\right)
\end{aligned}
$$

see also Abramowitz and Stegun (1965), Sections 17.4.15-16. Substituting the preceding results into the right hand side of Eq. 42 and redefining $\lambda:=$ $l / r \in(1, \infty)$, that is, replacing $\lambda$ with $1 / \lambda$, Eq. 22 is obtained.

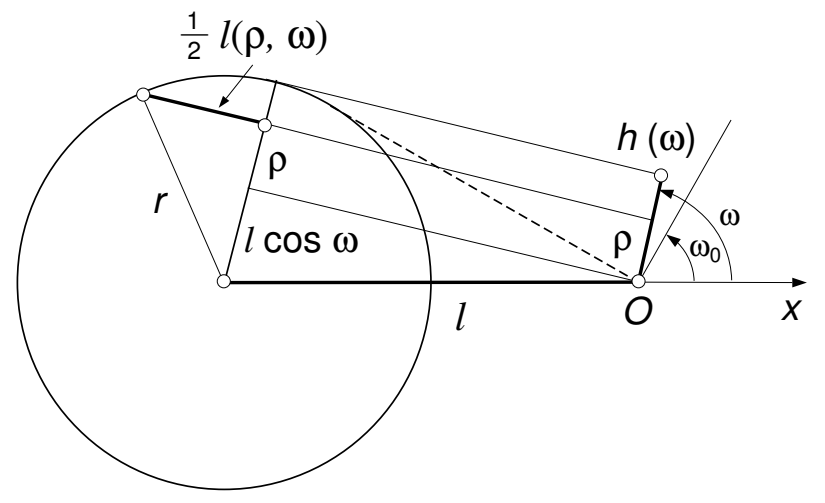

Fig. 8. Definitions used in the derivation of the mean wedge volume for a disk with exterior pivotal point $O$. 